compared to the published literature rates of individuals not receiving post-operative heparin. Additional variables included PTT range, type of heparin protocol used (weight based vs physician controlled), total time of heparinization, heparin dose, and number of PEDs deployed.

Results $0 \%(0 / 73)$ patients developed thrombotic complications in the post-operative period. The reported literature rate of symptomatic thrombotic events is $6.6 \%$. Post-operative heparin reduced symptomatic thrombotic complications following PED placement $(p=0.0125) .2 .7 \%(2 / 73)$ patients developed intraparenchymal hemorrhage resulting in neurological deficit, compared to a published rate of hemorrhagic complications approximating 3\%.

Conclusions Post-procedural low dose heparin prophylaxis reduces thrombotic complications in the post-operative period, without increasing hemorrhagic complications.

Disclosures I. Rybkin: None. J. Cooper: None. G. Kaur: None. F. Al-Mufti: None. C. Gandhi: None. J. Santarelli: None.

\section{P-033 DOES SYSTEMIC HYPERTENSION IMPACT RECANALIZATION OF COILED ANEURYSMS?}

Y Cho*. Radiology, Seoul National University Hospital, Seoul, Korea, republic of

\subsection{6/neurintsurg-2019-SNIS.69}

Background and Purpose It is well known that hypertension is a significant factor in the formation, growth, and rupture of aneurysms; and recanalization of coiled aneurysms is affected by hemodynamic stress. At present, however, the impact of hypertension on recanalization of coiled aneurysms has not been adequately investigated. We examined the relation between hypertension and subsequent outcomes of coiled aneurysms, using a matched patient analysis.

Methods A total of 715 subjects undergoing coil embolization of intracranial aneurysms between 2011 and 2013 were selected for study. Time-of-flight magnetic resonance (TOFMRA) or conventional angiography was used (singly or together) to gauge degrees of occlusion after coiling, applying the Raymond classification in grading recanalization. Patients with hypertension were grouped as controlled or uncontrolled, based on blood pressure (BP) readings at outpatient clinics. Hypertensive and non-hypertensive subjects were matched (1:1) for several relevant variables.

Results Overall, 484 patients (67.7\%) were hypertensive (controlled, 338; uncontrolled, 146). During the follow-up period (28.6 \pm 9.7 months), 129 aneurysms (18.0\%) displayed recanalization (minor, 58; major, 71). Patient age, concomitant diabetes, hyperlipidemia, aneurysm size, neck size, depth-to-neck ratio, and aneurysm type differed significantly in hypertensive and non-hypertensive groups. However, group incidences of cumulative recanalization were similar $(p=0.297)$. After 1:1 matching, the cumulative recanalization rate $(13.5 \%)$ in hypertensive and non-hypertensive counterparts $(14.3 \%)$ again proved similar $(p=0.578)$. In hypertensive group, in addition, recanalization showed no relation to controlled and uncontrolled subgroup ( $\mathrm{OR}=1.000, \mathrm{p}>0.999)$.

Conclusion Unlike other aspects of evolving aneurysms (ie, formation, growth, or rupture), recanalization of coiled aneurysms is seemingly unaffected by systemic hypertension.

Disclosures Y. Cho: None.

\section{P-034 4D DSA FOR SPINAL CORD VASCULAR MALFORMATIONS EXPLORATION: PRELIMINARY EXPERIENCE}

${ }^{1} \mathrm{~F}$ Clarençon*, 'S Lenck, ${ }^{1} \mathrm{E}$ Shotar, ${ }^{2} \mathrm{~A}$ Boch, ${ }^{1} \mathrm{~K}$ Premat, ${ }^{1} \mathrm{~J}$ Chiras, ${ }^{1} \mathrm{~N}$ Sourour. ${ }^{1}$ Neuroradiology, Pitié-Salpêtrière Hospital, Paris, France; ${ }^{2}$ Neurosurgery, Pitié-Salpêtrière Hospital, Paris, France

\subsection{6/neurintsurg-2019-SNIS.70}

Background and Purpose Spinal vascular malformations (SVMs) are aggressive diseases that may lead to neurological deficit due to spinal venous engorgement or secondary to hemorrhage.The endovascular treatment of such SVMs may be challenging with a significant risk of failure and/or a risk of severe neurological complications in case of spinal cord vascular supplies compromising. The precise understanding of the angio-architecture of SVMs is of tremendous importance to reduce the risk of treatment failure and the treatment-related complication risk.4D DSA (Siemens Healthcare) is a recently developed technology that has shown its potential for a better understanding of brain AVMs. The purpose of our study was to evaluate the potential of 4D DSA in spinal vascular malformations' exploration.

Material and methods Four consecutive patients (3 males, 1 female, mean age: $49 \pm 17$ y) with 5 spinal vascular malformations (spinal dural arteriovenous fistula [sDAVF]: $\mathrm{n}=2$, spinal pial arteriovenous fistula [sPAVF]: $\mathrm{n}=1$ and spinal arteriovenous malformations [sAVMS]: $\mathrm{n}=1$; one patient had 2 synchronous pial fistulas) had a spinal DSA including 4D DSA acquiiqisition.All the spinal DSA acquisitions were performed under general anesthesia. 4D DSA acquisitions were acquired with the protocol: '12sDSA Dyna4D Neuro'. $12 \mathrm{ml}$ of Iodixanol $320 \mathrm{mg}$ of $\mathrm{I} / \mathrm{ml}$ were injected via a $5 \mathrm{~F}$ catheter at $1 \mathrm{ml} / \mathrm{s}$ during the $12 \mathrm{~s}$ of the 4D DSA acquisition. The following criteria were evaluated in consensus by two reviewers:- overall quality of the acquisition- number of arterial feeders- location of the primary shunt point- venous drainage pattern: ascending, descending or both.

Results In $4 / 5$ of the cases ( 2 dural AV fistulas; 2 pial AV fistulas), the quality of the acquisition was graded good or fair. Satisfactory concordance between 4D DSA and the selective microcatheterization was observed in these 4 cases for the number of arterial feeders, the location of the shunt point and the venous drainage pattern. In one case of cervical spine AVM, the 4D DSA quality was graded poor and the angioarchitecture could not be satisfactorily analysed.

Conclusion 4D DSA acquisition may be helpful for a better understanding of spinal vascular malformations' angio-architecture. Larger series are warranted to confirm these first promising results.

Disclosures F. Clarençon: 2; C; Balt, Artedrone, Penumbra. S. Lenck: None. E. Shotar: None. A. Boch: None. K. Premat: None. J. Chiras: None. N. Sourour: 2; C; Medtronic.

\section{P-035 IS 3 YEARS ADEQUATE FOR TRACKING COMPLETELY OCCLUDED COILED ANEURYSMS?}

Y Cho*. Radiology, Seoul National University Hospital, Seoul, Korea, republic of

\subsection{6/neurintsurg-2019-SNIS.71}

Objective To ascertain the long-term durability of coiled aneurysms completely occluded at 36 months during follow-up given the potential for delayed recanalization. 